\title{
Droplet digital PCR-based circulating microRNA detection serve as a promising diagnostic method for gastric cancer
}

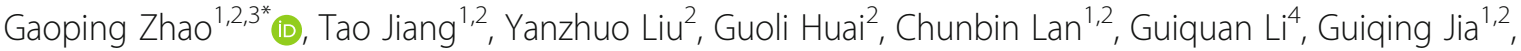 \\ Kang Wang ${ }^{1,2}$ and Maozhu Yang ${ }^{2,3,5^{*}}$
}

\begin{abstract}
Background: Novel non-invasive biomarkers for gastric cancer (GC) are needed, because the present diagnostic methods for GC are either invasive or insensitive and non-specific in clinic. The presence of stable circulating microRNAs (miRNAs) in plasma suggested a promising role as GC biomarkers.

Methods: Based on the quantitative droplet digital PCR (ddPCR), four miRNAs (miR-21, miR-93, miR-106a and miR-106b) related to the presence of GC were identified in plasma from a training cohort of 147 participants and a validation cohort of 28 participants.

Results: All circulating miRNA levels were significantly higher in the plasma of GC patients compared to healthy controls $(P<0.05)$. Through a combination of four miRNAs by logistic regression model, receiver operating characteristic (ROC) analyses yielded the highest AUC value of 0.887 in discriminating GC patients from healthy volunteers. Furthermore, miR21, miR-93 and miR-106b levels were significantly related to an advanced TNM stage in GC patients. ROC analyses of the combined miRNA panel also showed the highest AUC value of 0.809 in discriminating GC patients with TNM stage I and II from stage III and IV. Through combining four miRNAs and clinical parameters, a classical random forest model was established in the training stage. In the validation cohort, it correctly discriminated 23 out of 28 samples in the blinded phase (false rate, 17.8\%).
\end{abstract}

Conclusions: Using the ddPCR technique, circulating miR-21, miR-93, miR-106a and miR-106b could be used as diagnostic plasma biomarkers in gastric cancer patients.

Keywords: Gastric cancer, Liquid biopsy, ddPCR, miR-21, miR-93, miR-106a, miR-106b

\section{Background}

Gastric cancer is second most common cancer in terms of incidence and mortality in China, according to the most recent cancer statistics [1]. With the improvement of surgical technique, radiotherapy and chemotherapy in recent years, patients in the early stage of $\mathrm{GC}$ had a significant increased 5-year survival rate, but the prognosis for advanced GC remains poor [2, 3]. Thus, it is important to diagnose $\mathrm{GC}$ in the early stage thus yielding better outcome. Gastroscopy is the gold standard test for GC

\footnotetext{
* Correspondence: gzhao@uestc.edu.cn; 37771782@qq.com

1 Department of Gastrointestinal Surgery, Sichuan Academy of Medical Sciences \& Sichuan Provincial People's Hospital, Chengdu 610072, China ${ }^{2}$ School of Medicine, University of Electronic Science and Technology of China, Chengdu 610054, China

Full list of author information is available at the end of the article
}

diagnosis, but it is invasive and couldn't be frequently used as regular health examination. Carcinoembryonic antigen (CEA), $\alpha$-fetoprotein (AFP) and carbohydrate antigen 19-9 (CA19-9) are widely used as non-invasive markers in clinical, but their sensitivities and specificities are not enough for early diagnosis of GC [4]. Therefore, novel non-invasive biomarkers with better sensitivities and specificities are urgently needed.

MicroRNAs (miRNAs) are small noncoding RNAs, about 22-24 bases long, that inhibit their target mRNAs translation by inducing mRNA degradation or translational repression $[5,6]$. Up to now, there are thousands of miRNAs have been reported to be associated with tumor growth, invasion, metastasis and apoptosis [7]. Several studies have demonstrated that circulating

(c) The Author(s). 2018 Open Access This article is distributed under the terms of the Creative Commons Attribution 4.0 International License (http://creativecommons.org/licenses/by/4.0/), which permits unrestricted use, distribution, and reproduction in any medium, provided you give appropriate credit to the original author(s) and the source, provide a link to the Creative Commons license, and indicate if changes were made. The Creative Commons Public Domain Dedication waiver (http://creativecommons.org/publicdomain/zero/1.0/) applies to the data made available in this article, unless otherwise stated. 
miRNAs can serve as biomarkers for GC diagnosis. For example, miR-223, miR-16 and miR-100 were highly expressed in the serum of GC patients, and positively associated with TNM stage, metastatic status, tumor size and differentiation grade [8]. The level of let-7a expression in the plasma of GC patients was significant lower, and the value of the area under the receiver-operating characteristic curve was 0.879 for the miR-106a/let-7a ratio in GC patients and healthy volunteers [9]. Thus, miRNAs in peripheral blood have great potential for helping early diagnosis of GC.

Although the results of previous studies are promising, their clinical transferability remains uncertain, which mainly due to the lack of uniformity and reproducibility in the criteria for determining the circulating miRNA levels by quantitative real-time PCR (qPCR). Besides, several variables such as sample storage, RNA isolation, PCR inhibitors and normalization could affect final results [10]. The droplet digital PCR (ddPCR) technique is increasingly considered to be the gold standard in the application of liquid biopsy, because it has shown superior precision and sensitivity, being less affected by PCR inhibitors, and unnecessity of internal/external normalization while detecting low concentration of target nucleic acids molecules $[11,12]$. In this study, we used the ddPCR technique to explore the circulating miRNA signatures which could be potential biomarkers for GC diagnosis, and discriminating GC patients with different TNM stage. Four miRNAs, miR-21, miR-93, miR-106a and miR-106b, which have been most reported to be closely correlated with GC in tissue and plasma of patients and represent as candidate biomarkers for human GC, were examined by novel technique of ddPCR. [9, 13-15].

Furthermore, without the assist of tissue biopsy and imaging examinations, it would be difficult for clinicians to make diagnosis and tumor staging for $\mathrm{GC}$, because there are many factors could probably influence the results. To improve the precision and accuracy of diagnosing disease, new approaches such as machine learning which is the main technical basis for data mining, provide an effective solution [16]. Several studies have been reported to use machine learning tools for data mining to diagnose disease or predict prognosis [17-19]. In this study, we explored the use of random forest model based learning for GC diagnosis, by using circulating miRNA expressions and clinical parameters such as age, gender, CEA and CA19-9.

\section{Methods}

\section{Patients and blood samples}

The present study was approved by the ethics committee of Sichuan Provincial People's Hospital. All participants provided written informed consent form to approve the use of their blood samples for research purposes.

From Sichuan Provincial People's Hospital, a total of 101 patients with gastric cancer (GC) and 46 healthy volunteers were recruited to the training cohort between January 2017 and June 2017, and a total of 11 patients with GC and 17 healthy volunteers were recruited to the validation cohort between December 2017 and February 2018. For plasma, $5 \mathrm{ml}$ peripheral blood was collected in EDTA tubes, the sampling time was pre-surgery for GC patients, especially. And within $2 \mathrm{~h}$, plasma was separated by centrifugation at $2000 \times \mathrm{g}$ for $10 \mathrm{~min}$, the supernatant was followed by a second centrifugation at $12000 \times \mathrm{g}$ for $20 \mathrm{~min}$. Then, the plasma was either stored at $-80^{\circ} \mathrm{C}$ or miRNA was extracted immediately.

For patients, GC paraffin-embedded tissue samples were obtained after surgical resection. The clinicopathological classification and staging were determined according to the World Health Organization pathological classification of tumors. The clinical information for GC patients in the training stage is summarized in Table 1. Among the 101 patients included 51 male and 50 female, the median age was 56 years old (range, 35-75 years) and the median tumor size was $3.9 \mathrm{~cm}$ (range, 1.0-7.5 cm). There were 16 cases well differentiated, 35 were moderately differentiated and 50 were poorly differentiated. There were 35 cases without lymph node metastasis, 66 cases with lymph node metastasis, 18 cases with distant metastasis and 83 cases without distant metastasis. According to TNM stage classification, 28 cases were categorized as stage I, 13 cases for stage II, 36 cases for stage III and 24 cases for stage IV.

\section{RNA isolation and reverse transcription}

Total circulating miRNA was extracted from $200 \mu \mathrm{L}$ plasma using the miRNeasy Serum/Plasma Kit (Qiagen) according to the manufacturer's protocol. In addition, $10 \mu \mathrm{L}$ of a $1.5 \mathrm{nmol} / \mathrm{L}$ solution of the custom synthetic miRNA cel-miR-54-5p was added after the sample was mixed with $1 \mathrm{~mL}$ QIAzol Lysis reagent for $5 \mathrm{~min}$. RNA was eluted from spin columns in $40 \mu \mathrm{L}$ nuclease-free water.

Four circulating human miRNAs (miR-21, miR-93, miR-106a and miR-106b) and one spike-in control miRNA (cel-miR-54-5p) were determinated by TaqMan ${ }^{\text {tw }}$ MicroRNA Assays, TaqMan miRNA Reverse Transcription kits (Life Technologies) and miRNA-specific RT primers were used for reverse transcription. For each sample, $3 \mu \mathrm{L}$ RNA sample was added in a $15 \mu \mathrm{L}$ reaction mixture using standard protocol. Then, the resulting cDNA was prepared for the droplet digital PCR.

\section{ddPCR workflow}

For each ddPCR assay, $3 \mu \mathrm{L}$ cDNA sample, $10 \mu \mathrm{L} 2 \times$ ddPCR supermix for probes (Bio-Rad), $1 \mu \mathrm{L} 20 \times$ TaqMan miRNA probe and $6 \mu \mathrm{L}$ RNase-free Water was 
Table 1 Clinicopathological characteristics of all individuals in the training stage and relationships with circulating miRNAs in the plasma

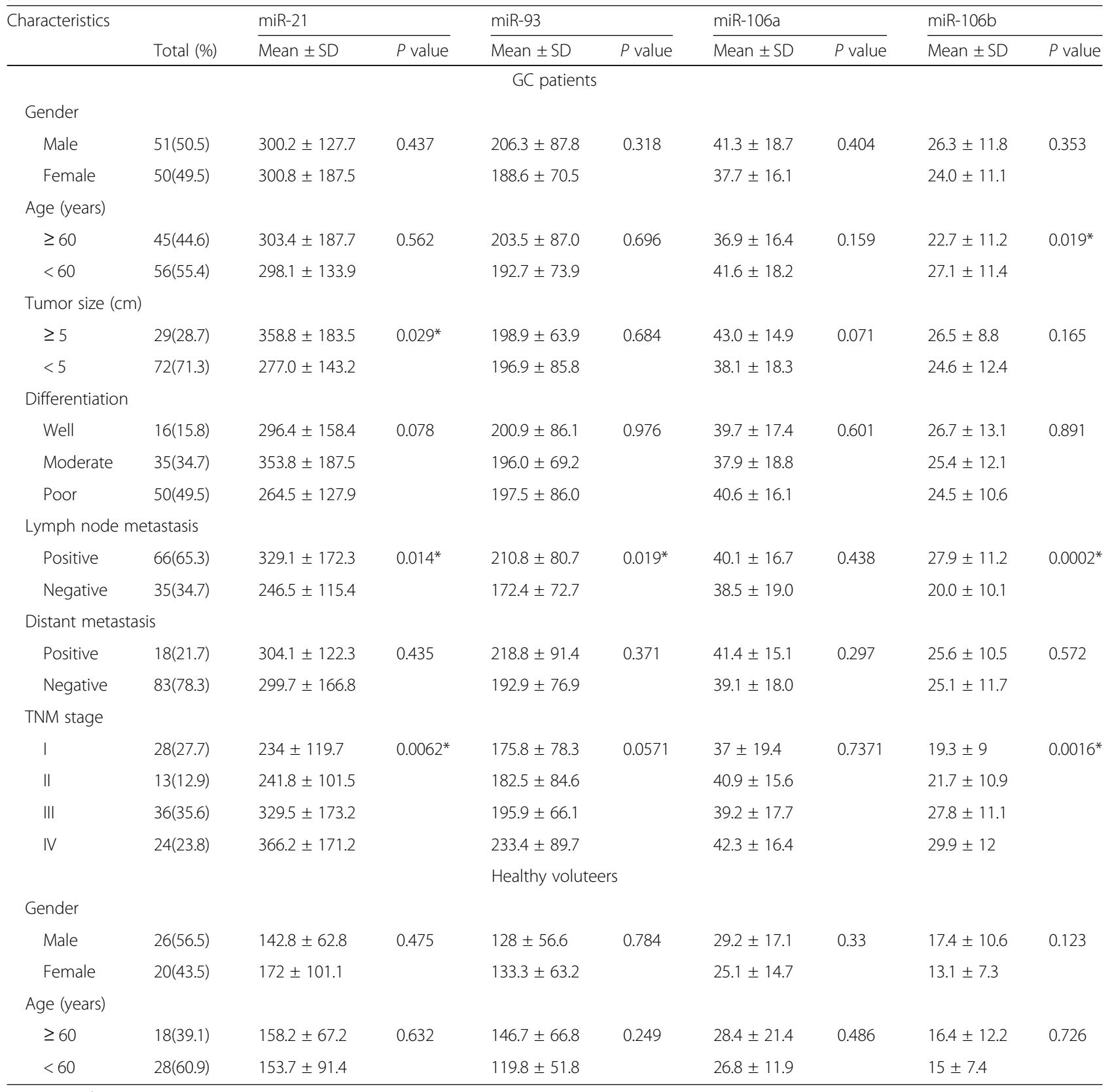

*means $P$-value $<0.05$

added in a $20 \mu \mathrm{L}$ reaction mixture. Then, the mixture and $70 \mu \mathrm{L}$ droplet generation oil for probes (Bio-Rad) were respectively loaded into the sample wells and oil wells of a disposable droplet generator cartridge (Bio-Rad). After that, droplets were generated by QX200 droplet generator device (Bio-Rad) and carefully transferred to a 96-well PCR plate (Eppendorf). The cycling conditions were: $95{ }^{\circ} \mathrm{C}$ for $10 \mathrm{~min}, 40$ cycles of $95{ }^{\circ} \mathrm{C}$ for $15 \mathrm{~s}$ and $57{ }^{\circ} \mathrm{C}$ for $1 \mathrm{~min}$, and a final step at $98^{\circ} \mathrm{C}$ for $10 \mathrm{~min}$. At the end of the PCR reaction, droplets were read in the QX200 droplet reader and analyzed using the Quantasoft ${ }^{\mathrm{tm}}$ version 1.7.4 software (Bio-Rad). In addition, a no template control (NTC) was included in every assay. And the spike-in control miRNA was used as an internal calibrator to monitor extraction efficiency.

\section{Statistical analysis}

The statistical analyses were performed using the SPSS version 19.0 software. The Mann-Whitney U test was used to compare significant differences in 
miRNA expression between different groups. Logistic regression was used to develop a combined miRNA panel to diagnose GC with different TNM stage. Receiver operating characteristic (ROC) curves were established to evaluate the capacity of the tested miRNA to discriminate cancer cases in different TNM stage, and its potential use as a diagnostic tool for detecting GC. A $p$-value of less than 0.05 was considered to be significant.

A total of 147 participants in the training cohort were grouped into the training data set, and 28 participants in the validation cohort were grouped into the testing data set. In the training stage, a classical random forest algorithm in $\mathrm{R}$ version 3.4.2 software was used to construct variable selection models for combined four miRNA panel and clinical parameters in this study. Next, using single blind method, we tested the model by using the 28 cases of the testing data set as a prospective validation set, to assess its predictive ability. And we also retrospectively analyzed the 147 cases of the training data set.

\section{Results}

Circulating miRNAs in plasma of GC patients versus healthy controls

First, we compared the expression levels of four validated miRNAs in plasma from healthy volunteers $(n=46)$ and GC patients $(n=101)$ with different TNM stage using ddPCR. All four miRNAs including miR-21, miR-93, miR-106a and miR-106b levels were significantly lower in healthy controls than GC patients with TNM stage I ( $p=0.0021, p=0.0084$, $p=0.0116$ and $p=0.0168$ respectively) (Fig. 1a), as well as TNM stage II, III and IV (Table 2). To evaluate the diagnostic value of the concentrations of these four circulating miRNAs, ROC curve analysis was performed. GC patients with different TNM stage were combined as one group, the area under the curve (AUC) values of miR-21, miR-93, miR-106a and miR-106b were 0.811 (95\% confidence interval [CI], 0.739-0.884), 0.751 (95\% CI, 0.667-0.836), 0.731 (95\% CI, $0.638-0.823)$ and 0.77 (95\% CI, 0.683-0.857), respectively (Fig. 1b). We also detect the CEA and CA19-9 in all 147 participants in the present study, the testing time was pre-surgery for GC patients. The AUC values obtained for

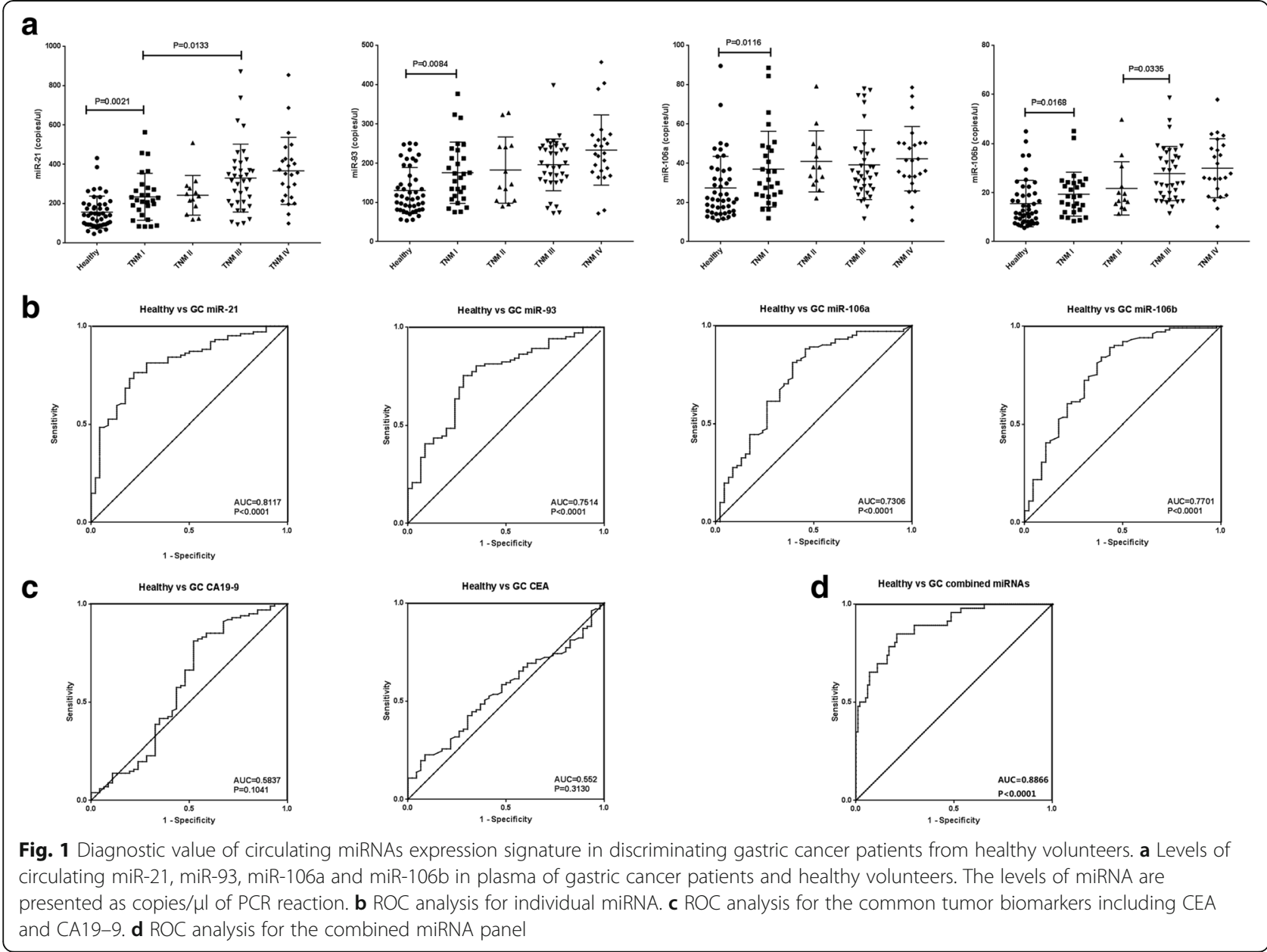


Table 2 Performance of circulating miRNAs for detection of GC with different TNM stages

\begin{tabular}{lllll}
\hline & $\begin{array}{l}\text { miR-21 } \\
\text { P-value }\end{array}$ & miR-93 & miR-106a & miR-106b \\
\hline Health vs GC TNM I & $0.0021^{* *}$ & $0.0084^{* *}$ & $0.0116^{*}$ & $0.0168^{*}$ \\
Health vs GC TNM II & $0.0014^{* *}$ & $0.0263^{*}$ & $0.0013^{* *}$ & $0.0132^{*}$ \\
Health vs GC TNM III & $<0.0001^{* *}$ & $<0.0001^{* *}$ & $0.0004^{* *}$ & $<0.0001^{* *}$ \\
Health vs GC TNM IV & $<0.0001^{* *}$ & $<0.0001^{* *}$ & $0.0001^{* *}$ & $<0.0001^{* *}$ \\
GC TNM I VS II & 0.5704 & 0.8142 & 0.2820 & 0.6043 \\
GC TNM I VS III & $0.0133^{*}$ & 0.1543 & 0.4864 & $0.0018^{* *}$ \\
GC TNM I VS IV & $0.0018^{* *}$ & $0.0102^{*}$ & 0.1118 & $0.0004^{* *}$ \\
GC TNM II VS III & 0.0791 & 0.4665 & 0.5262 & $0.0335^{*}$ \\
GC TNM II VS IV & $0.0163^{*}$ & 0.0952 & 0.6097 & $0.0200^{*}$ \\
GC TNM III VS IV & 0.2985 & 0.0897 & 0.2164 & 0.3092 \\
\hline$*$ means P< 0.05,** mean & $P<0.01$ & & &
\end{tabular}

* means $P<0.05$, ** means $P<0.01$

CEA and CA19-9 to distinguish the GC patients from the healthy controls were $0.552(95 \% \mathrm{CI}, 0.456-0.648)$ and 0.584 (95\% CI, 0.473-0.695), respectively (Fig. 1c).

Furthermore, through a combination of the expression levels of four validated miRNAs, weighted by the regression coefficient, we developed a miRNA classifier using logistic regression model. It could be used to evaluate the predicted probability of being detected as GC, which was calculated as follows: first, the expression levels of four miRNAs were calculated as miRNA panel score using the following equation: miRNA panel score= 5.218-0.011 × miR-21-0.012 × miR-93-0.037 ×

miR-106a-0.031 $\times$ miR-106b. Then, the predicted probability was calculated by a second equation: predicted probability $=$ EXP (miRNA panel score) $/[1+$ EXP (miRNA panel score)]. The combination of the four miRNAs exhibited better diagnostic value compared to any individual miRNA, with an AUC of 0.887 (95\% CI, $0.83-0.943$ ) (Fig. 1d) by logistic regression analysis, an optimal cut-off point was indicated at 0.315 with a sensitivity of $84.8 \%$ and a specificity of $79.2 \%$. These results indicated that the circulating miR-21, miR-93, miR-106a and miR-106b could be considered as more accurate biomarkers than CEA and CA19-9 for GC diagnosis.

\section{Circulating miRNAs in plasma of GC patients with different TNM stage}

Although four miRNAs were up-regulated in GC patients with TNM stage II compared with stage I, as well as TNM stage IV compared with stage III, but the differences had no statistically significant $(p>0.05)$ (Fig. 1a \& Table 2). However, miR-21 and miR-106b levels were significantly increased in GC patients with TNM stage III or IV compared with stage I (miR-21: $p=0.0133$ and $p=0.0018$, miR-106b: $p=0.0018$ and $p=0.0004$ respectively) (Table 2). miR-106b levels were still significantly increased when compared TNM stage III or IV with stage II in GC patients $(p=0.0335$ and $p=0.02$ respectively), while it was significant for miR-21 levels only when compared TNM stage IV with stage I in GC patients $(p=0.0163)$. Moreover, miR-93 and miR-106a levels in GC patients had no significant difference between groups with different TNM stage, except for the miR-93 levels in GC patients with TNM stage I and IV $(p=0.0102)$.

Furthermore, we combined GC patients with TNM stage I and II as one group, as well as TNM stage III and IV. As expected, the results showed that miR-21 and miR-106b levels were significantly higher in stage III and IV compared to stage I and II $(p=0.0004$ and $p<0.0001$ respectively), while miR-106a levels still had no significant difference between these two groups $(p=0.3824)$ (Fig. 2a). However, there was an unexpected significant increase for miR-93 levels in GC patients with stage III and IV compared to stage I and II $(p=0.0218)$ (Fig. 2a). The AUC values obtained for miR-21, miR-93, miR-106a and miR-106b to distinguish the GC patients with stage I and II from stage III and IV were 0.704 (95\% CI, 0.601-0.807), 0.634 (95\% CI, 0.52-0.749), 0.552 (95\% CI, 0.435-0.668) and 0.736 (95\% CI, 0.635-0.836), respectively (Fig. 2b).

Same as previous analysis, we assigned each patient a risk score which was calculated as follows: First, miRNA panel score $=-2.875+0.005 \times$ miR-21 $+0.003 \times$ miR-93-0.034 $\times$ miR-106a $+0.115 \times$ miR-106b. Then, risk score $=\mathrm{EXP}$ (miRNA panel score)/[1 + EXP (miRNA panel score)]. The combination of the four miRNAs exhibited better capability to discriminate GC with TNM stage I and II from stage III and IV compared to any individual miRNA, with an AUC of 0.809 (95\% CI, 0.723-0.896) (Fig. 2c) by logistic regression analysis, an optimal cut-off point was indicated at 0.534 with a sensitivity of $78.3 \%$ and a specificity of $70.7 \%$. Taken together, these results demonstrated that circulating miR-21, miR-93 and miR-106b might have a potential diagnostic value for distinguishing GC with different TNM stage.

\section{Correlation between expression levels of circulating miRNA in plasma and clinicopathologic factors in GC patients}

Except for the TNM stage, we evaluated whether the levels of four circulating miRNAs are correlated with other clinical characteristics of all GC patients. As it was summarized in Table 1 , the expression of miR-21, miR-93, miR-106a and miR-106b didn't significantly differ between the GC patients based on gender $(p=0.437, p=0.318, p=0.404$ and $p=0.353$ respectively), tumor differentiation ( $p=0.078, p=0.976, p$ $=0.601$ and $p=0.891$ respectively) and distant metastasis ( $p=0.435, p=0.371, p=0.297$ and $p=0.572$ respectively). 

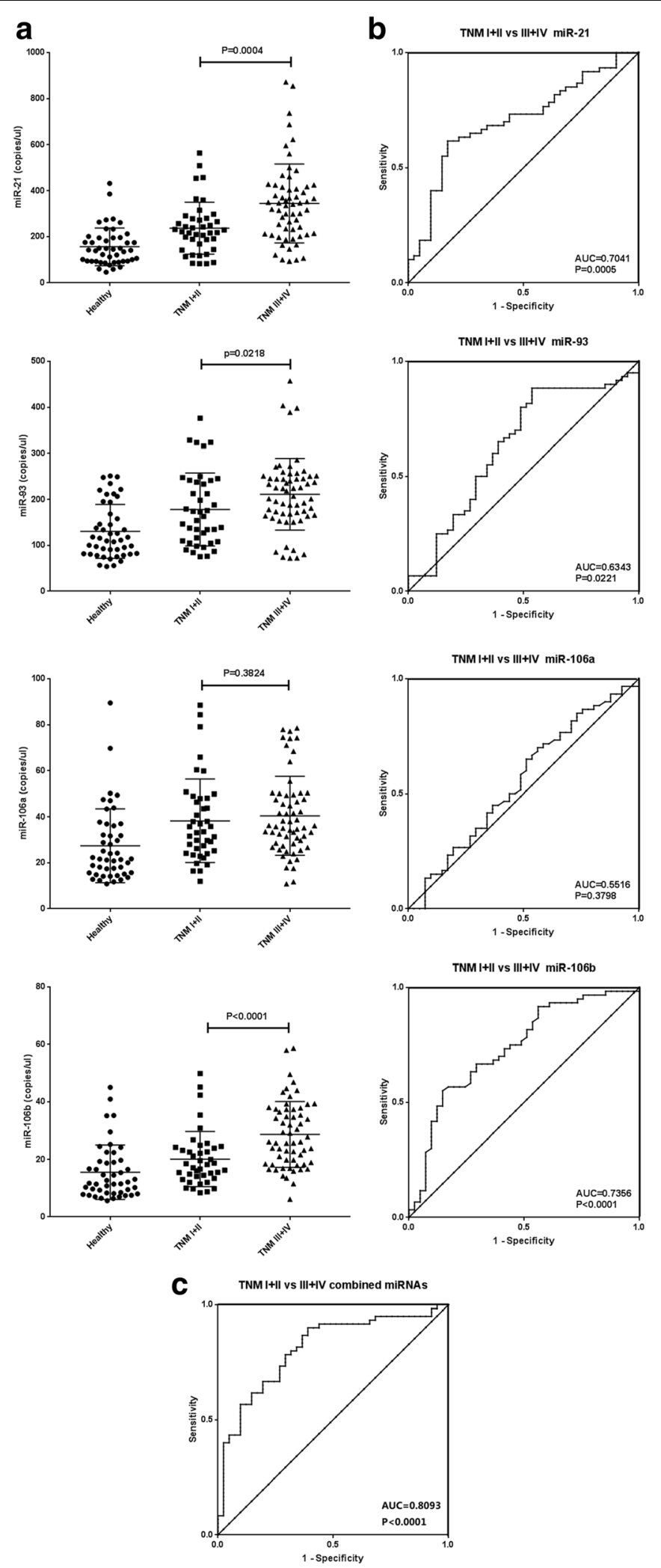

Fig. 2 (See legend on next page.) 
(See figure on previous page.)

Fig. 2 Diagnostic value of circulating miRNAs expression signature in discriminating gastric cancer at different TNM stage. a Levels of circulating miR-21, miR-93, miR-106a and miR-106b in plasma of gastric cancer patients with low TNM stage (stage I and II) and high TNM stage (stage III and IV), and healthy volunteers. The levels of miRNA are presented as copies/ $\mu \mathrm{l}$ of PCR reaction. $\mathbf{b}$ ROC analysis for individual miRNA. c ROC analysis for the combined miRNA panel

However, the levels of miR-21, miR-93 and miR-106b in plasma from the GC patients is significantly related to lymph node metastasis $(p=0.014, p=0.019$ and $p=0.0002$ respectively). Moreover, the circulating miR-21 and miR-106b levels are also related to tumor size and age respectively $(p=0.029$ and $p=0.019)$. The results demonstrated the feasibility of these miRNAs for the diagnosis of other clinicopathological characteristics of GC patients.

\section{Random forest model used for GC diagnosis}

To evaluate the diagnostic value of circulating miR-21, miR-93, miR-106a and miR-106b combined with other conventional clinical parameters including gender, age, CEA and CA19-9 for GC with different TNM stage, we used a classical random forest algorithm for analysis. A total of 147 participants in the training cohort were grouped as the training data set and used for developing the model. And the other 28 participants in the validation cohort were grouped as the testing data set and used for assessing predictive ability of the model.

In the training stage, using random forest supervised classification algorithm, four microRNAs, three clinical parameters including CEA, age and CA19-9 mostly related to the diagnostic classification were selected (Additional file 1: Figure S1). In the testing stage, we used the developed random forest model to validate both the training data set and the testing data set. It correctly discriminated 147 out of 147 samples in the training data set, and 23 out of 28 samples in the testing data set, which showed 100 and $82.1 \%$ accuracy respectively in identifying Healthy volunteers and GC patients with different TNM stage (Table 3), by using the selected variables based on their value cut-offs (Additional file 1: Figure S1). In addition, the most influential factor in this model was miR-21, followed by miR-106b, miR-93, miR-106a, CA19-9, CEA, age and gender (Table 4).

\section{Discussion}

Early diagnosis could greatly improve the survival rates of GC patients. However, the currently used diagnostic methods are either invasive or insensitive, thus limited their application in clinic. In recent years, a number of circulating miRNAs, which are notably stable in the circulation of body fluids [20,21], are suggested as promising non-invasive diagnostic markers for GC [9, 15, 22-24]. Unfortunately, since circulating miRNAs exist in blood at extremely low concentrations [25], the test results would be made poorly repeatable due to the interference of several variables, such as sample processing protocols, RNA isolation and so on $[10,26]$. Most importantly, quantitative real-time PCR is most commonly used but must rely on the use of external calibrators, because it lacks reliable endogenous reference miRNA for normalization of results in plasma or serum. Therefore, the data which produced by a variety of normalization methods in different studies, become non-comparable or difficult to compare. This is a major obstacle for their translation into clinically useful applications [10, 27].

The present study, to our best knowledge, is the first to evaluate the diagnostic value of circulating miRNAs for GC patients using the ddPCR technique. ddPCR is a recently introduced technology which can achieve absolute quantification of nucleic acids based on the principles of sample portioning, end-point PCR and Poisson statistics $[28,29]$. Thus, it overcomes the normalization and calibrator issues [30]. Besides, it has shown better precision and sensitivity while detecting low concentration of target nucleic acids molecules [31, 32]. More importantly, ddPCR can tolerate PCR inhibitors which could influence the efficiency of PCR amplification, without affecting the quantitative results of the target [11].

Using ddPCR, we analyzed the levels of circulating miR-21, miR-93, miR-106a and miR-106b in the plasma of GC patients and healthy volunteers. Similar to previous studies $[9,14,15]$, we found the significantly increased levels of these miRNAs in GC patients compared with healthy controls, and some miRNAs were associated with advanced TNM stage. ROC curve analysis showed that each miRNA had higher diagnostic

Table 3 Confusion matrix of the developed random forest model in the testing stage

\begin{tabular}{|c|c|c|c|c|}
\hline & & \multicolumn{3}{|c|}{ Predicted class } \\
\hline & & Healthy & TNM I + II & TNM III + IV \\
\hline \multicolumn{5}{|c|}{ Training data set } \\
\hline \multirow[t]{3}{*}{ Actual class } & Healthy & 46 & 0 & 0 \\
\hline & TNM I + II & 0 & 41 & 0 \\
\hline & TNM III + IV & 0 & 0 & 60 \\
\hline \multicolumn{5}{|c|}{ Testing data set } \\
\hline \multirow[t]{3}{*}{ Actual class } & Healthy & 14 & 2 & 1 \\
\hline & TNM I + II & 0 & 4 & 1 \\
\hline & TNM III + IV & 1 & 0 & 5 \\
\hline
\end{tabular}


Table 4 The relative importance of variables in the developed random forest model

\begin{tabular}{ll}
\hline Variables & Mean Decrease Gini \\
\hline Gender & 1.134787 \\
Age & 9.342937 \\
CA19-9 & 11.051689 \\
CEA & 9.918123 \\
miR-21 & 19.143754 \\
miR-93 & 16.274413 \\
miR-106a & 12.627928 \\
miR-106b & 16.550531 \\
\hline
\end{tabular}

sensitivity and specificity than CEA and CA19-9 which were widely used in clinic. Furthermore, through a combination of the expression levels of four validated miRNAs, a patient will be considered to have GC if the predicted probability is higher than the threshold set ( 0.315 with a sensitivity of $84.8 \%$ and specificity of $79.2 \%$ ) in the model. An AUC of 0.887 (95\% CI, 0.83-0.943) and $P$-values $<0.001$ indicate the great potential value of these miRNAs as $\mathrm{GC}$ biomarkers.

Based on the results above, we further evaluate the potential use of these miRNAs in discriminating GC with different TNM stage. First, GC patients with TNM stage I and II were combined as one group, as well as stage III and IV, because there was no statistically significant difference between these groups. Then, our results showed that the levels of circulating miR-21, miR-93 and miR-106b in the plasma of GC patients were significantly higher in TNM stage III and IV than stage I and II, except for the miR-106a. As usual, a combination of four miRNAs showed better capability to discriminate GC with different TNM stage. A patient will be considered to have GC with TNM stage III or IV if the risk score is higher than 0.534 (a sensitivity of $78.3 \%$ and specificity of 70.7\%). ROC analysis also showed an AUC of 0.809 (95\% CI, 0.723-0.896) and P-values $<0.001$. To our knowledge, this study is the first to demonstrate that these miRNAs might be also used as biomarkers to discriminate GC with TNM stage I and II from stage III and IV.

In the search of possible correlations with clinicopathological features, it was noteworthy that the presence of lymph node metastases was significantly correlated with increased levels of circulating miR-21, miR-93 and miR-106b. Moreover, a high level of circulating miR-21 was significantly related to a bigger tumor size $(\geq 5 \mathrm{~cm})$. These results indicate that these miRNAs might represent biomarkers of tumor aggressiveness, which further improved their value for discriminating GC with different TNM stage. Some studies have reported that high levels of miR-21 expression may induce tumor proliferation, migration and invasion via the downregulation of Noxa or PTEN expressions in GC cells [33, 34]. And miR-93 could promote proliferation and metastasis of $\mathrm{GC}$ via targeting TIMP2 or inactivation of the Hippo signaling pathway [35, 36]. In cancer-associated fibroblasts from GC, miR-106b could promote cell migration and invasion by targeting PTEN [37]. And it could also promote cell cycling of GC cells through regulation of p21 and E2F5 target gene expression [38]. These might be the mechanism of its correlation with lymph node metastases and tumor size. However, although it was reported that miR-106a could also regulate invasion and metastasis of $\mathrm{GC}$ via targeting TIMP2 [39, 40], and may inhibit extrinsic apoptotic pathway through targeting FAS [41], our results demonstrated that miR-106a expression was not associated with the lymph node metastases and tumor size. Further studies are required.

In clinic, due to the numerous factors that influence the precision and accuracy of diagnosing diseases or predicting of patients' prognosis, more and more studies are applying machine learning algorithms to medical data, including the detection of GC [20, 42, 43]. There are several algorithms such as random forest, support vector machine and neural networks were commonly used $[43,44]$. Here, we chose random forest model since it is easy to interpret, and allowed us to estimate the importance of a variable. After the random forest model was established in the training stage, when we tested the predictive value of this model using the testing data set, our results showed that it correctly discriminated 14 out of 17 healthy volunteers (false rate, 17.6\%), 4 out of 5 GC patients with TNM stage I or II (false rate, 20\%), and 5 out of $6 \mathrm{GC}$ patients with TNM stage III or IV (false rate, 16.7\%). However, the number of cases included in the present study is still far from sufficient to develop a reliable model, and we also didn't have enough cases to test and validate the model. Further studies with much more cases are urgently required, to improve their application in clinic. Moreover, despite our results and accumulating evidences suggested that circulating miRNAs stably existed in circulation and can indeed be used as biomarkers to identify and monitor a variety of cancers and other diseases, it is still unknown how and why GC causes changes in the levels of these four circulating miRNAs, and whether or how they play roles in physiology. Further studies are also needed.

\section{Conclusions}

Overall, the present study demonstrated that by using the ddPCR technique, circulating miR-21, miR-93, miR-106a and miR-106b could be used as diagnostic plasma biomarkers in gastric cancer patients. 


\section{Additional files}

Additional file 1: Figure S1. A random forest model for discriminating healthy volunteers, gastric cancer patients with low TNM stage (stage I and II) and high TNM stage (stage III and IV) G0 represents healthy volunteers; G1 represents GC patients with TNM stage I and II; G2 represents GC patients with TNM stage III and IV. (TIF 624 kb)

\section{Abbreviations}

AFP: a-fetoprotein; AUC: Area under the curve.; CA19-9: Carbohydrate antigen 19-9; CEA: Carcinoembryonic antigen; ddPCR: Droplet digital PCR; GC: Gastric cancer; miRNA: Microrna; qPCR: Quantitative real-time PCR; ROC: Receiver operating characteristic

\section{Funding}

This study was supported by the grants from the National Natural Science Foundation of China (No.81172832, 81771723), Sichuan Youth Science and Technology Foundation (No.2013JQ0020), Special Program for Sichuan Youth Science and Technology Innovation (No.2014TD0010), and grant from Health and Family Planning Commission of Sichuan Province (No.110190). The funding body did not have any role in the study design, nor the data collection, analysis and interpretation as well as writing of the manuscript.

\section{Availability of data and materials}

The datasets used and/or analysed during the current study are available from the corresponding author on reasonable request.

\section{Authors' contributions}

The authors' contribution was as following: GPZ: Conceptualization, methodology, formal analysis, investigation, resources, data curation, funding acquisition, writing-original draft, writing-review and editing, and project administration. TJ, YZL and GLH: Validation, formal analysis, investigation, resources, and data curation. CBL and GQL: Conceptualization, formal analysis. GQJ and KW: Conceptualization, methodology, formal analysis. MZY: Conceptualization, methodology, funding acquisition, writing-original draft, writing-review and editing. All authors read and approved the final manuscript.

\section{Ethics approval and consent to participate}

The present study was approved by the ethics committee of Sichuan Provincial People's Hospital. All patients provided written informed consent.

\section{Consent for publication}

Not applicable

\section{Competing interests}

The authors declare that they have no competing interests.

\section{Publisher's Note}

Springer Nature remains neutral with regard to jurisdictional claims in published maps and institutional affiliations.

\section{Author details \\ ${ }^{1}$ Department of Gastrointestinal Surgery, Sichuan Academy of Medical Sciences \& Sichuan Provincial People's Hospital, Chengdu 610072, China. ${ }^{2} \mathrm{~S} c h o o l$ of Medicine, University of Electronic Science and Technology of China, Chengdu 610054, China. ${ }^{3}$ Institute of Chengdu Biology, and Sichuan Translational Medicine Hospital, Chinese Academy of Sciences, Chengdu 610041, China. ${ }^{4}$ Department of General Surgery, Qionglai Medical Center Hospital, Chengdu, Sichuan Province, China, Chengdu 611530, China. ${ }^{5}$ Department of General Surgery, Sichuan Academy of Medical Sciences \& Sichuan Provincial People's Hospital, Chengdu 610072, China.}

Received: 2 April 2018 Accepted: 15 June 2018

Published online: 22 June 2018

\section{References}

1. Siegel R, Naishadham D, Jemal A. Cancer statistics, 2013. CA Cancer J Clin. 2013;63:11-30.
2. Hamashima C, Shabana M, Okada K, Okamoto M, Osaki Y. Mortality reduction from gastric cancer by endoscopic and radiographic screening. Cancer Sci. 2015;106:1744-9.

3. Catalano V, Labianca R, Beretta GD, Gatta G, de Braud F, Van Cutsem E. Gastric cancer. Crit Rev Oncol Hematol. 2009;71:127-64.

4. Pasechnikov V, Chukov S, Fedorov E, Kikuste I, Leja M. Gastric cancer: prevention, screening and early diagnosis. World J Gastroenterol. 2014;20:13842-62.

5. Bartel DP. MicroRNAs: target recognition and regulatory functions. Cell. 2009;136:215-33.

6. Filipowicz W, Bhattacharyya SN, Sonenberg N. Mechanisms of posttranscriptional regulation by microRNAs: are the answers in sight? Nat Rev Genet. 2008;9:102-14.

7. Di Leva G, Garofalo M, Croce CM. MicroRNAs in cancer. Annu Rev Pathol. 2014;9:287-314.

8. Wang H, Wang L, Wu Z, Sun R, Jin H, Ma J, et al. Three dysregulated microRNAs in serum as novel biomarkers for gastric cancer screening. Med Oncol. 2014:31:298.

9. Tsujiura M, Ichikawa D, Komatsu S, Shiozaki A, Takeshita H, Kosuga T, et al. Circulating microRNAs in plasma of patients with gastric cancers. $\mathrm{Br} \mathrm{J}$ Cancer. 2010;102:1174-9.

10. Jarry J, Schadendorf D, Greenwood C, Spatz A, van Kempen LC. The validity of circulating microRNAs in oncology: five years of challenges and contradictions. Mol Oncol. 2014:8:819-29.

11. Racki N, Dreo T, Gutierrez-Aguirre I, Blejec A, Ravnikar M. Reverse transcriptase droplet digital PCR shows high resilience to PCR inhibitors from plant, soil and water samples. Plant Methods. 2014;10:42.

12. Campomenosi $P$, Gini $E$, Noonan DM, Poli A, D'Antona P, Rotolo N, et al. A comparison between quantitative PCR and droplet digital PCR technologies for circulating microRNA quantification in human lung cancer. BMC Biotechnol. 2016:16:60.

13. Ueda T, Volinia S, Okumura H, Shimizu M, Taccioli C, Rossi S, et al. Relation between microRNA expression and progression and prognosis of gastric cancer: a microRNA expression analysis. Lancet Oncol. 2010;11:136-46.

14. Tchernitsa O, Kasajima A, Schäfer R, Kuban RJ, Ungethüm U, Györffy B, et al. Systematic evaluation of the miRNA-ome and its downstream effects on mRNA expression identifies gastric cancer progression. J Pathol. 2010;222:310-9.

15. Shiotani A, Murao T, Kimura Y, Matsumoto H, Kamada T, Kusunoki H, et al. Identification of serum miRNAs as novel non-invasive biomarkers for detection of high risk for early gastric cancer. Br J Cancer. 2013;109:2323-30.

16. Cios KJ, Moore GW. Uniqueness of medical data mining. Artif Intell Med. 2002;26:1-24.

17. Kang J, Schwartz R, Flickinger J, Beriwal S. Machine learning approaches for predicting radiation therapy outcomes: a Clinician's perspective. Int J Radiat Oncol Biol Phys. 2015;93:1127-35.

18. Sudharsan B, Peeples M, Shomali M. Hypoglycemia prediction using machine learning models for patients with type 2 diabetes. J Diabetes Sci Technol. 2015:9:86-90.

19. Miyaki R, Yoshida S, Tanaka S, Kominami Y, Sanomura Y, Matsuo T, et al. A computer system to be used with laser-based endoscopy for quantitative diagnosis of early gastric cancer. J Clin Gastroenterol. 2015;49:108-15.

20. Armand-Labit V, Pradines A. Circulating cell-free microRNAs as clinical cancer biomarkers. Biomol Concepts. 2017;8:61-81.

21. Wittmann J, Jäck HM. Serum microRNAs as powerful cancer biomarkers. Biochim Biophys Acta. 2010;1806:200-7.

22. Shin VY, Ng EK, Chan WW, Kwong A, Chu KM. A three-miRNA signature as promising non-invasive diagnostic marker for gastric cancer. Mol Cancer. 2015;14:202.

23. Zhou X, Zhu W, Li H, Wen W, Cheng W, Wang F, et al. Diagnostic value of a plasma microRNA signature in gastric cancer: a microRNA expression analysis. Sci Rep. 2015;5:11251.

24. Tsujiura M, Komatsu S, Ichikawa D, Shiozaki A, Konishi H, Takeshita H, et al. Circulating miR-18a in plasma contributes to cancer detection and monitoring in patients with gastric cancer. Gastric Cancer. 2015;18:271-9.

25. Wang K, Yuan Y, Cho JH, McClarty S, Baxter D, Galas DJ. Comparing the MicroRNA spectrum between serum and plasma. PLoS One. 2012;7:e41561.

26. Sourvinou IS, Markou A, Lianidou ES. Quantification of circulating miRNAs in plasma: effect of preanalytical and analytical parameters on their isolation and stability. J Mol Diagn. 2013:15(6):827-34.

27. Marzi MJ, Montani F, Carletti RM, Dezi F, Dama E, Bonizzi G, et al. Optimization and standardization of circulating MicroRNA detection for clinical application: the miR-test case. Clin Chem. 2016;62:743-54. 
28. Pinheiro LB, Coleman VA, Hindson CM, Herrmann J, Hindson BJ, Bhat S, et al. Evaluation of a droplet digital polymerase chain reaction format for DNA copy number quantification. Anal Chem. 2012;84:1003-11.

29. Huggett JF, Foy CA, Benes V, Emslie K, Garson JA, Haynes R, et al. The digital MIQE guidelines: minimum information for publication of quantitative digital PCR experiments. Clin Chem. 2013;59:892-902.

30. Zhao H, Wilkins K, Damon IK, Li Y. Specific qPCR assays for the detection of orf virus, pseudocowpox virus and bovine papular stomatitis virus. J Virol Methods. 2013;194:229-34.

31. Zhao S, Lin H, Chen S, Yang M, Yan Q, Wen C, et al. Sensitive detection of porcine circovirus-2 by droplet digital polymerase chain reaction. J Vet Diagn Investig. 2015;27:784-8.

32. Brunetto GS, Massoud R, Leibovitch EC, Caruso B, Johnson K, Ohayon J, et al. Digital droplet PCR (ddPCR) for the precise quantification of human Tlymphotropic virus 1 proviral loads in peripheral blood and cerebrospinal fluid of HAM/TSP patients and identification of viral mutations. J NeuroOncol. 2014;20:341-51.

33. Sun H, Wang P, Zhang Q, He X, Zai G, Wang X, et al. MicroRNA-21 expression is associated with the clinical features of patients with gastric carcinoma and affects the proliferation, invasion and migration of gastric cancer cells by regulating Noxa. Mol Med Rep. 2016;13(3):2701-7.

34. Zhang BG, Li JF, Yu BQ, Zhu ZG, Liu BY, Yan M. microRNA-21 promotes tumor proliferation and invasion in gastric cancer by targeting PTEN. Oncol Rep. 2012;27(4):1019-26.

35. Guan H, Li W, Li Y, Wang J, Li Y, Tang Y, et al. MicroRNA-93 promotes proliferation and metastasis of gastric cancer via targeting TIMP2. PLOS One. 2017;12(12):e0189490.

36. Li L, Zhao J, Huang S, Wang Y, Zhu L, Cao Y, et al. MiR-93-5p promotes gastric cancer-cell progression via inactivation of the hippo signaling pathway. Gene. 2018;641:240-7

37. Yang TS, Yang XH, Chen X, Wang XD, Hua J, Zhou DL, et al. MicroRNA-106b in cancer-associated fibroblasts from gastric cancer promotes cell migration and invasion by targeting PTEN. FEBS Lett. 2014;588(13):2162-9.

38. Yao YL, Wu XY, Wu JH, Gu T, Chen L, Gu JH, et al. Effects of microRNA-106 on proliferation of gastric cancer cell through regulating p21 and E2F5. Asian Pac J Cancer Prev. 2013;14(5):2839-43.

39. Zhu M, Zhang N, He S, Yan R, Zhang J. MicroRNA-106a functions as an oncogene in human gastric cancer and contributes to proliferation and metastasis in vitro and in vivo. Clin Exp Metastasis. 2016;33(5):509-19.

40. Zhu M, Zhang N, He S, Lui Y, Lu G, Zhao L. MicroRNA-106a targets TIMP2 to regulate invasion and metastasis of gastric cancer. FEBS Lett. 2014;588(4):600-7.

41. Wang Z, Liu M, Zhu H, Zhang W, He S, Hu C, et al. miR-106a is frequently upregulated in gastric cancer and inhibits the extrinsic apoptotic pathway by targeting FAS. Mol Carcinog. 2013;52(8):634-46.

42. Liu WZ, White AP, Hallissey MT, Fielding JW. Machine learning techniques in early screening for gastric and oesophageal cancer. Artif Intell Med. 1996:8:327-41.

43. Li Q, Wang W, Ling X, Wu JG. Detection of gastric cancer with Fourier transform infrared spectroscopy and support vector machine classification. Biomed Res Int. 2013;2013:942427.

44. Kuruvilla J, Gunavathi K. Lung cancer classification using neural networks for CT images. Comput Methods Prog Biomed. 2014;113:202-9.

\section{Ready to submit your research? Choose BMC and benefit from:}

- fast, convenient online submission

- thorough peer review by experienced researchers in your field

- rapid publication on acceptance

- support for research data, including large and complex data types

- gold Open Access which fosters wider collaboration and increased citations

- maximum visibility for your research: over $100 \mathrm{M}$ website views per year

At BMC, research is always in progress.

Learn more biomedcentral.com/submissions 